“C 2017 IEEE. Personal use of this material is permitted. Permission from IEEE must be obtained for all other uses, in any current or future media, including reprinting/republishing this material for advertising or promotional purposes, creating new collective works, for resale or redistribution to servers or lists, or reuse of any copyrighted component of this work in other works." 


\title{
Investigations of a Load-Bearing Composite Electrically Small Egyptian Axe Dipole Antenna
}

\author{
Thomas C. Baum, Member, IEEE, Richard Ziolkowski, Fellow, IEEE, Kamran Ghorbani, Member, \\ IEEE and Kelvin J. Nicholson, Member, IEEE
}

\begin{abstract}
An electrically small, metamaterial-inspired Egyptian Axe Dipole (EAD) antenna has been investigated for use in structural composite materials. The EAD antenna consists of a differentially-fed dipole element integrated with a near-field resonant parasitic (NFRP) EAD element. These elements have been adapted to these materials resulting in a system that is impedance matched and radiates efficiently at $307 \mathrm{MHz}$. Three cases have been identified and investigated to ascertain the performance of the manufacturing techniques and material properties used to build these electrically small antennas (ESAs), as well as their performance characteristics. Uniquely, an embroidered conductive thread and a new carbon fiber based, non-woven mat have been investigated for use as the conducting elements. Both cases are compared with a copper variant of the EAD antenna. All three prototypes were tested. Measurements confirm that both the non-woven mat and the embroidered versions of the EAD antennas perform similarly, with maximum realized gains ranging from $1.72-1.90 \mathrm{dBi}$.
\end{abstract}

Index Terms-composite structures, electrically small antennas, embroidered textiles, metamaterial-inspired antennas, pregtronics

\section{INTRODUCTION}

$\mathrm{M}$ ULTi-FunCtion, conformal, load-bearing, antennas structures (CLAS) provide the unique capability of offering structural weight savings in composite aerospace platforms [1]-[9]. New load-bearing antennas, such as the Slotted-Waveguide Antenna Stiffened Structure (SWASS), have shown the benefits of merging electromagnetic systems and composite structure designs [3], [6], [7], [9]. Despite it being a highly complex antenna structure to manufacture, SWASS has many practical benefits in its topology for high frequency phased array radar applications [6]. However, many

Manuscript received January 07, 2017. This work was supported by the Australian Department of Defence, Defence Science and Technology Group (DST Group) SRI-SME.

T. C. Baum and K. Ghorbani are with the School of Electrical and Computer Engineering, Royal Melbourne Institute of Technology (RMIT University), Melbourne, Victoria, Australia, 3001 (e-mail: thomas.baum@rmit.edu.au, kamran.ghorbani@rmit.edu.au).

K. J. Nicholson is with the Defence Science and Technology Group at Fisherman's Bend, Melbourne, Victoria, Australia, 3207

R. W. Ziolkowski is with the University of Technology Sydney, Global Big Data Technologies Centre, Ultimo NSW 2000, Australia, and the University of Arizona, Department of Electrical and Computer Engineering, 1230 E. Speedway Blvd., Tucson, AZ 85721, USA (e-mail: Richard.Ziolkowski@uts.edu.au) aircraft communications systems fall within the HF to UHF frequency bands. Efficient antennas in these ranges are physically large and are generally found in the form of blade/surface monopole and/or lone wire antennas. The art of designing metamaterial-inspired antennas has seen many of these large antennas down-sized to geometries approaching the $\mathrm{Chu} /$ Wheeler limit, while still maintaining respectable radiation efficiencies [10], [11]. However, with a decrease in an antennas size, there is a direct trade-off in the antennas' impedance bandwidth. This has recently been overcome with the introduction of active, non-Foster elements in the form of negative impedance converters (NICs) and related active devices (e.g., [12]-[14]). Doubling on these advances are Huygens source electrically small antennas (ESAs) which radiate in their broadside direction (e.g., [15]-[18]).

With the advent of these new antenna technologies, the ability to seamlessly integrate passive and active ESA antennas into aerospace platforms is becoming increasingly important [1]-[9]. These antennas have many key advantages, including the capability to be downsized for integration into small aerospace platforms. This is of particular importance in the case of smaller unmanned aerial systems (UAS) where there are significant internal volume and weight restrictions.

Recent advances in the integration of novel conductors and active devices into composite materials have made it possible to realize actively tunable NICs for conformal loadbearing antennas structures (CLAS) [19]-[21]. Thus, if one could also integrate an ESA into such composite materials, then their broadband versions are immediately possible.

Based on textile technologies, the Pregtronics concept allows for a viable avenue to develop active ESA in composite materials [19]. The embroidered threads and the integration of non-woven carbon fiber veil materials can be considered to develop such structures. These materials, however, are considered to be lossy conductors at microwave frequencies. This is especially the case for the non-woven carbon fiber veil materials [20]. Hence, the use of these lossy conductors could result in poor radiation efficiency of ESAs. This reduced radiation efficiency could be further accentuated by the high field strengths found within and near these ESAs.

This paper presents an investigation of the integration of an ESA, the so-called Egyptian Axe Dipole (EAD) antenna [22], [23], into load-bearing composite materials. In particular, it was integrated into a sandwiched, quartz-fiber reinforced polymer matrix (QFRP). The conductive radiating near-field 
resonant parasitic (NFRP) EAD elements and their driven dipole elements have been integrated into the panel using two methods. The first is a textile approach, using the embroidery of a conductive thread (Aracon XS0200E-025) to sew the antenna components into the composite material. The second is a new, lightweight, laser-cut, copper-coated non-woven carbon veil manufactured by TFP and modified in-part by the Australian Defence, Science and Technology Group (DST Group). Both of these methods have been analyzed for their ability to provide reasonable antenna performance while remaining compatible with existing aerospace composite manufacturing techniques. It should also be noted that this work significantly extends the authors initial work presented in [21].

\section{EAD ANTENNA DESIGN}

The antenna is based on a top-loaded EAD NFRP element arranged with additional arms and coupled with a driven meander dipole element. Due to this loading condition, the topology of the EAD NFRP element presented here is related, but not identical to the topology of previous EAD dipole realizations [12], [13], [17], [18], [22], [23]. To reduce the substrate losses and to make the EAD NFRP antenna compatible with aerospace composite manufacturing processes, a core stiffened panel was proposed. This structure is often referred to as a sandwich panel because it consists of two fiber reinforced polymer (FRP) composite skins with a central lightweight core structure. This arrangement provides significant stiffness to the consolidated panel substrate.

The core materials used in sandwich composites vary dramatically based on their application. They can range from lightweight wood to honeycomb structures made of paper and metals (such as Nomex). More recently, lightweight, high density, closed-cell foams have become popular due to their flexibility in resin processing techniques. The closed-cell form of the foam also reduces their moisture uptake caused by atmospheric diffusion. This property is ideal for antenna applications as moisture introduces high losses into any material. These losses would have a significant effect on the radiation efficiency of ESAs over their life span. Rohacell HF foam was chosen for the core structure of the manufactured EAD antennas. This material has been processed successfully up to $140^{\circ} \mathrm{C}$ under vacuum conditions, which is higher than that recommended on its datasheet. The core thickness for all of the EAD antenna designs was chosen to be $3.0 \mathrm{~mm}$. The design may be altered to suit any core thickness so long as it satisfies the field coupling requirements for exciting the EAD NFRP component.

The following measured or known material properties have been used for the simulation of the EAD antennas: cyanate ester resin/astroquartz II composite $(\varepsilon=3.42+0.02 \mathrm{i}$ at 300 $\mathrm{MHz})$, Rohacell HF Foam $(\varepsilon=1.0449+0.00021 \mathrm{i}$ at $300 \mathrm{MHz})$, copper shim $\left(\sigma=5.8 \times 10^{7} \mathrm{~S} / \mathrm{m}\right)$, Aracon XS0200E-025 thread ( $Z=0.026$ ohms/sq. [19]) and the copper veil $(Z=0.0067$ ohms/sq.). It should be noted that the surface resistance of the

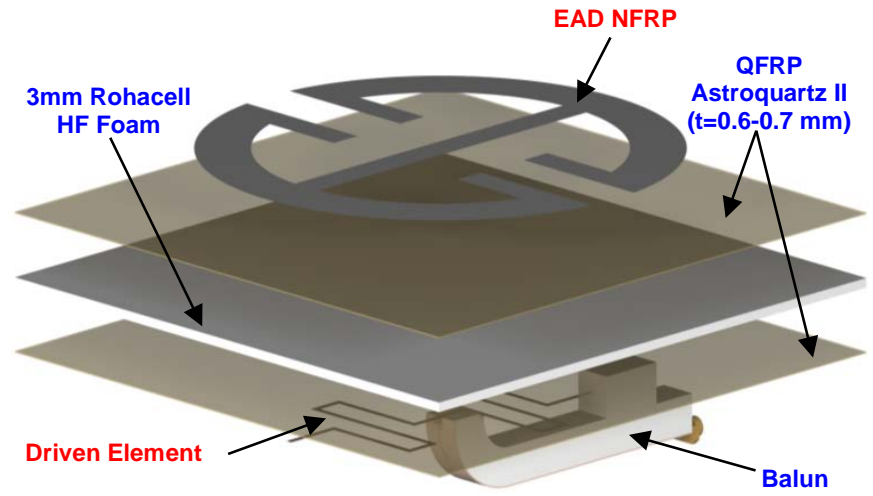

Fig. 1. A 3D exploded view of the EAD showing its construction layers.

copper veil was measured using a transmission line method similar to that outlined in [19]. The QFRP dielectric was measured using full transmission reflection coaxial air-line Nicholson-Ross-Weir extraction method [24].

Both the Aracon XS0200E-025 thread and the copper veil were assumed to be isotropic conductors in the simulation models. Both materials were also assumed to have a thickness $>0.15 \mathrm{~mm}$ to meet the skin depth requirements at $300 \mathrm{MHz}$. The overall planar dimensions of the EAD substrate were fixed at $180 \mathrm{~mm}$ x $180 \mathrm{~mm}$. A total of nine plies of Astroquatz II fabric were used to build the QFRP substrate, resulting in an ideal consolidated thickness of between 0.6 and $0.7 \mathrm{~mm}$ ( $\sim 0.073 \mathrm{~mm}$ per ply). Figure 1 gives a general 3D overview of the antenna's topology.

The initial dimensions and shapes of the EAD NFRP and driven elements were selected from previous works (i.e. [13]). Because the conductivity was high for both materials, this seemed to be a good choice; it was not expected to have a significant effect on the resonance frequency of the antenna. Based on the proposed substrate configuration shown in Fig. 1 and the knowledge of those existing EAD topologies, the initial design of the EAD antenna was set at $300 \mathrm{MHz}(\lambda=$ $1.0 \mathrm{~m})$.

The EAD antenna was first designed and simulated with CST Microwave Studio using an ideal feeding condition at $50 \Omega$ input impedance. It was found that the substrate materials impacted the resonance frequency, and the design had to be modified. While the EAD NFRP element initially had thin trace widths, they were increased to provide some inductive reactance. The additional arms on the NFRP were introduced to tailor the reactance: the size of the resulting gap modifies the capacitance while the arm length modifies the inductance. The most significant modification was the introduction of meander elements into the driven dipole element to increase its inductive reactance. This meanderapproach had been successful in previous NFPR element designs [22] and was necessary here because of the change in the capacitance realized by the sandwich substrate. The EAD antenna was then optimized for best matching at $300 \mathrm{MHz}$ by varying the length of the meander and capacitive are lengths.

Traditionally the driving element of EAD antennas has been directly fed with an SMA connector. To remove the need for a 
SMA connector and any interference from the feeding coaxial cable on the radiation pattern, a truncated tapered microstrip balun was introduced to differentially feed the antenna.

\section{A. Balun Feed Geometry}

The truncated tapered microstrip balun was selected to excite the driven element in all three EAD cases. This balun was designed to match the $50 \Omega$ source to the $50 \Omega$ input impedance of the EAD antennas. Previous feeding arrangements for EAD antennas which used direct feeding from SMA/Coaxial connectors can be observed in [12], [13], [17], [18], [22], [23]. While it worked well for engineered substrate materials such as Rogers Duroid ${ }^{\mathrm{TM}} 5880$, the differential feed became necessary because of the layered material arrangement of the feeding structure and those of the driven and NFRP elements. A direct SMA/coaxial feeding approach for the composite versions of the EAD antenna was determined to be more complex due to the possibility of large manufacturing variability. The balun choice was made despite the fact that a larger differential feeding structure could impact the overall radiation performance of the antenna. The latter occurs because of the long wavelength of the low operating frequency which facilitates strong coupling of the balun structure to the driven and NFRP elements.

To achieve a balanced feed, a truncated tapered microstrip balun suspended on a Rohacell HF foam support was designed. The balun is the required $50 \Omega$ to $50 \Omega$ transition that tapers a microstrip transmission line (MSL) to a differential parallel strip line (PSL). The MSL-to-PSL transition was designed on Rogers 5880, 0.508mm (0.02in) thick substrate having a $17 \mu \mathrm{m}(0.5 \mathrm{oz}$.) copper thickness. The balun's dimensions and simulated S-parameter values are presented in Figs. 2 and 3, respectively. Fig. 3c gives the dimensions of the Rohacell HF foam support, which was bonded to the antenna. Each balun was soldered to the driven element and a radial SMA connector soldered to the MSL.

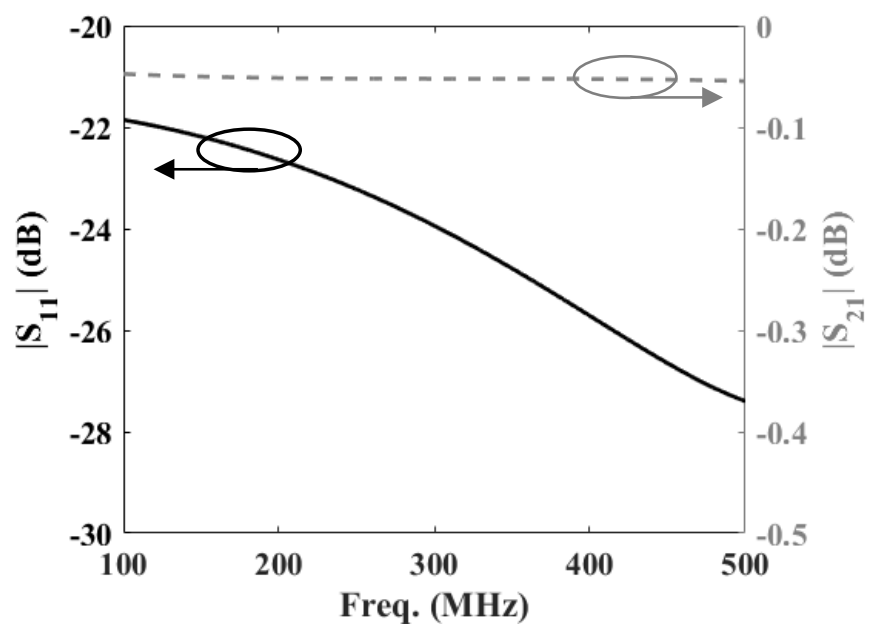

Fig. 2. Simulated S-parameters as functions of the source frequency for the truncated tapered microstrip-to-parallel-strip balun.

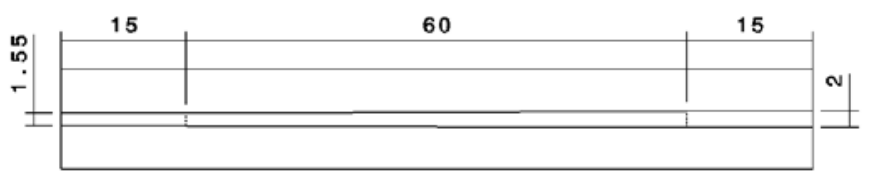

(a)

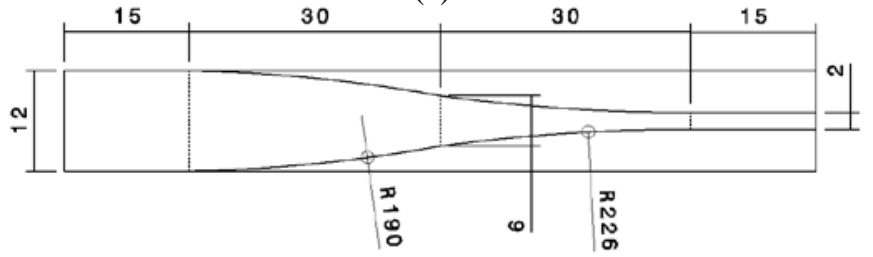

(b)

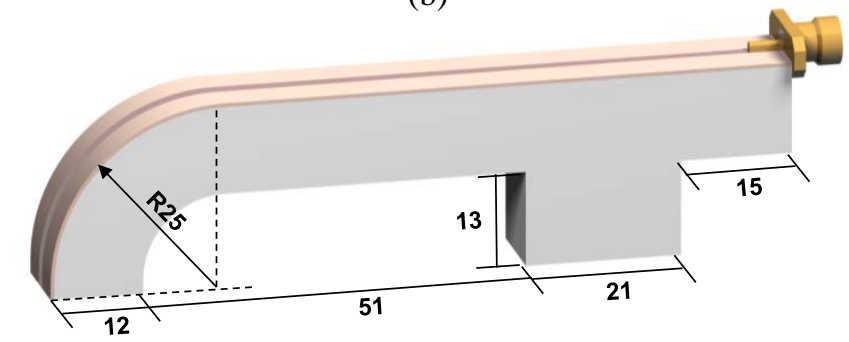

(c)

Fig. 3. Dimensions (mm) of the balun design. a) Parallel strip line transition, b) ground structure, and c) optimized Rohacell HF foam-based feed support structure.

\section{B. EAD Geometry}

The introduction of this balun feeding structure affected the tuning of the EAD. Optimal matching for the complete structure was found to occur at $307 \mathrm{MHz}$. The final EAD dimensions are illustrated in Fig. 4. The magnitudes of the simulated reflection coefficient $\left(\left|S_{11}\right|\right)$ for all three EAD antennas as a function of the source frequency are presented in Fig. 5a.

The reflection coefficient for the driven meander-modified dipole element alone has also been included in Fig. 5a to emphasize the attained impedance matching. By itself, a near perfect port reflection occurred at the end of the balun. This result highlights the fact that the driven element is not a standalone radiating element at the design frequency. As Fig. 5 a illustrates, the simulations predicted that with the addition of the EAD NFRP element, all three antennas demonstrated very good impedance matching at a resonance frequency of $307 \mathrm{MHz}$. The reactance values for changes in the capacitive arm length (l arm) and drive arm length (l drive arm) are illustrated in Figs. 5b and Fig. 5c, respectively. These have been included to show what effects the two geometric features have on the tuning of the EAD structure, as well as their resultant reactance (capacitive or inductive).

It should be noted that the slightly smaller input reflection coefficients obtained for the Aracon XS0200E-025 thread and the copper veil illustrated in Fig. 5a are due to differences in the conductive losses, not improvements in the radiation efficiency. The loss issue is highlighted by where the maximum E-field intensity distributions occur in the system. They are illustrated in Fig. 6 on the front and back sides of the copper shim EAD antenna. 

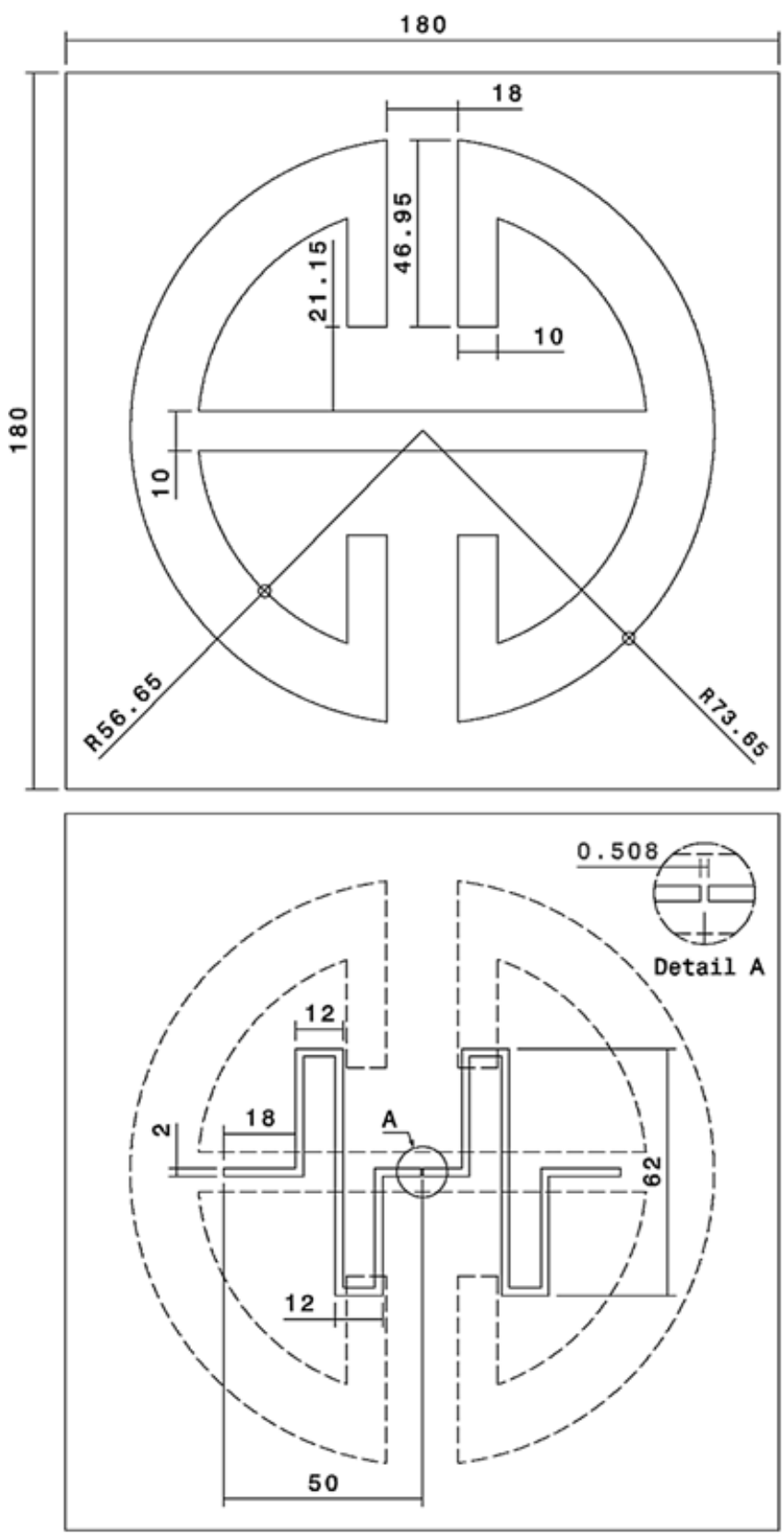

Fig. 4. Overview of the finalized EAD design showing both the EAD NFRP element (top) and the driven element (bottom) that achieved a $50 \Omega$ input impedance match to the source (dimensions in $\mathrm{mm}$ ).

The E-field behavior is highly concentrated near the capacitive arms of the EAD NFRP element and the meander portions of the driven element. Another feature of the E-field intensity distributions to note is its higher concentration around the perimeter of the EAD NFRP element. It must be noted that both the conductive and dielectric losses within these areas will then have the greatest effects on the radiation efficiency in all cases. The embroidered Aracon XS0200E-025 thread is inherently anisotropic by nature. Any anisotropy will force the fields near a thread to be capacitively coupled to neighboring threads, resulting in an effectively higher conductive loss [20].

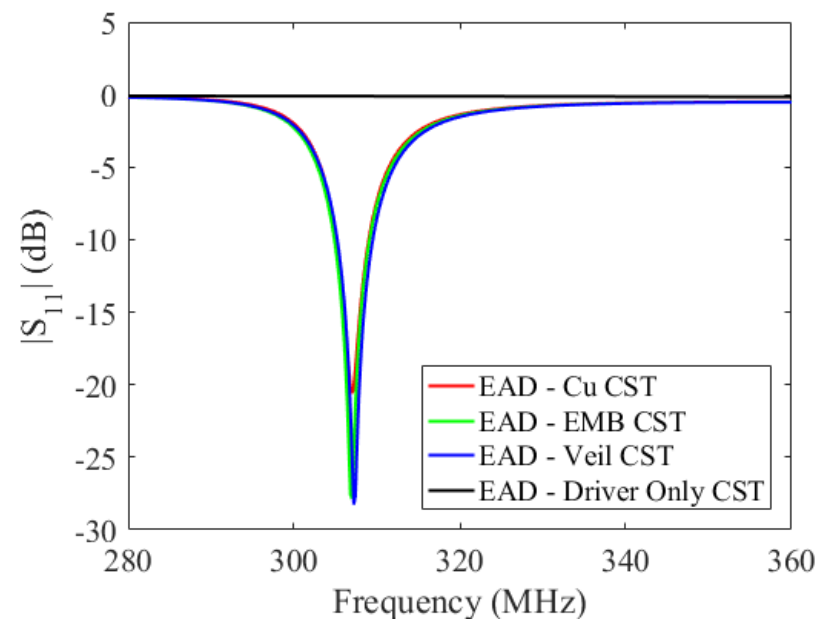

(a)

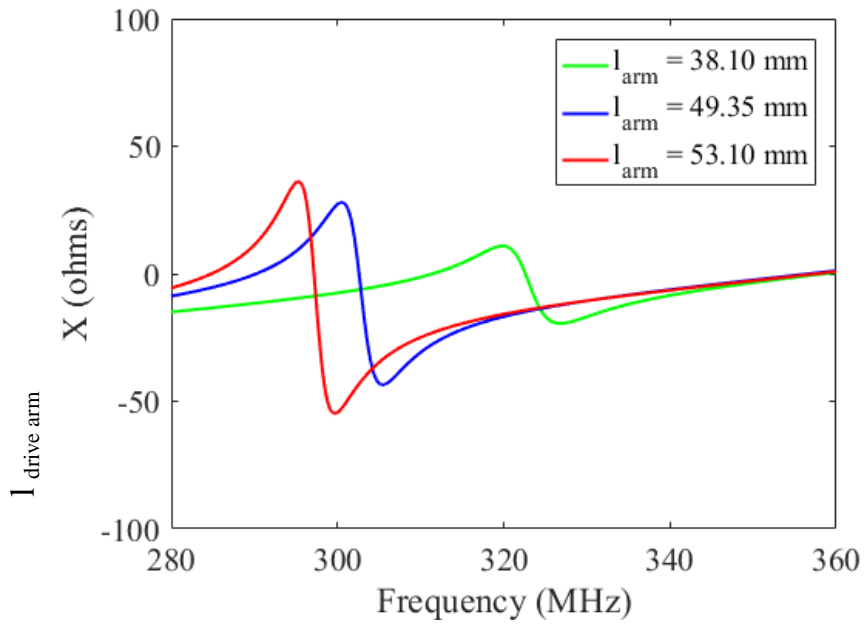

(b)

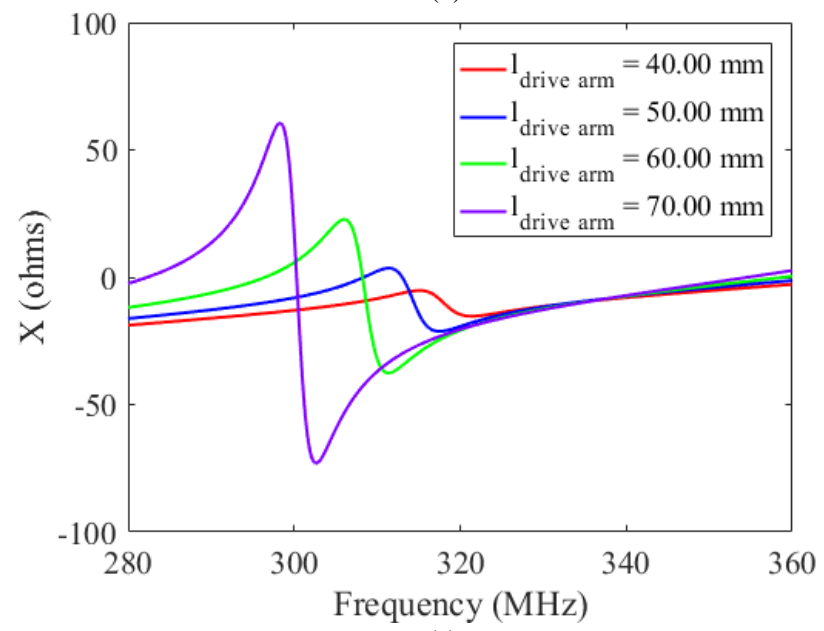

(c)

Fig. 5. Simulated (a) $\left|S_{11}\right|$ values, (b) reactance values when the capacitive arm length of the EAD NFRP element is increased, and (c) reactance values when the meander arm length of the driven element is increased, versus the source frequency for all three EAD antennas and the driven element alone. 

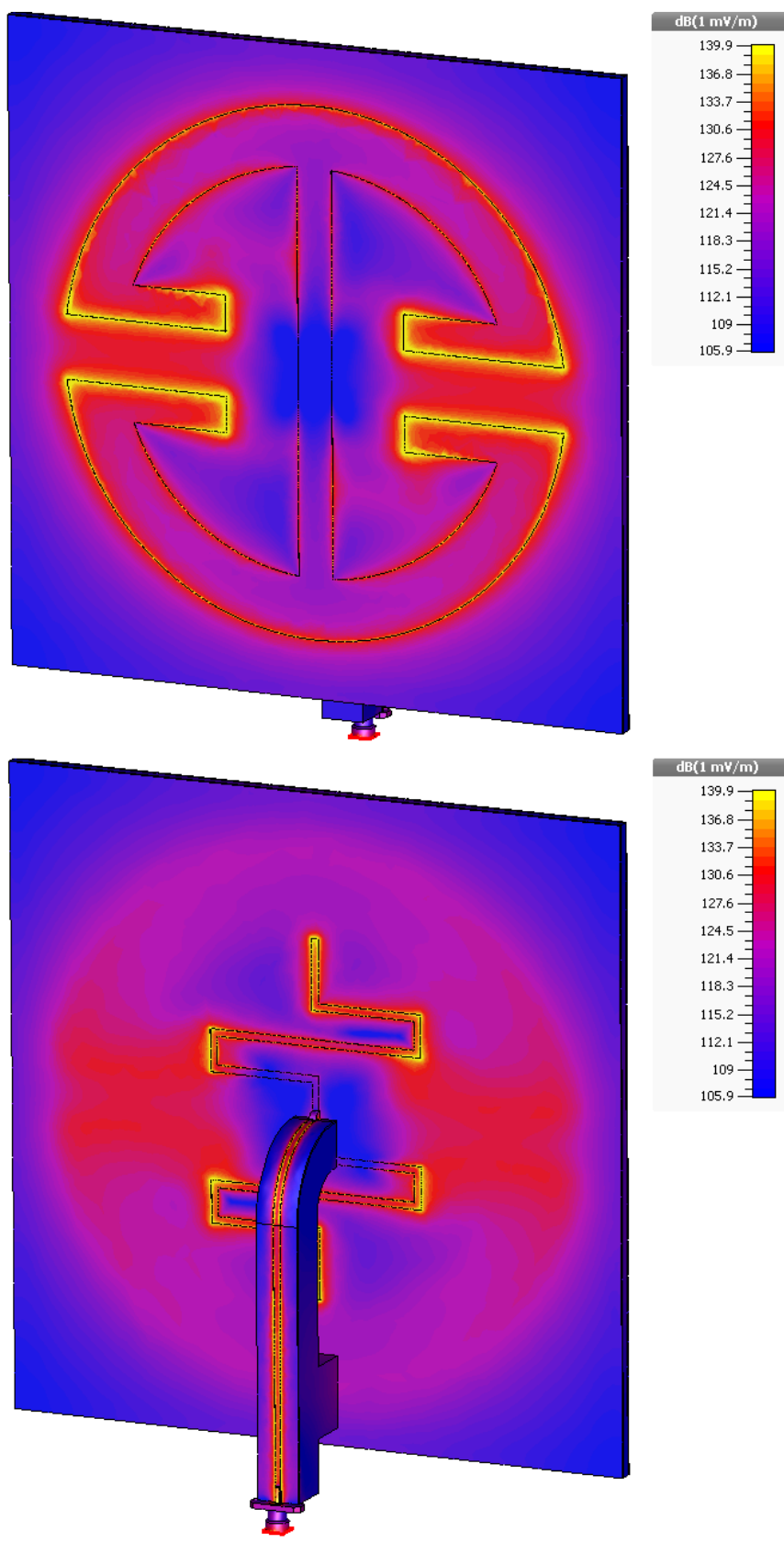

Fig. 6. Simulated E-field intensity plots of the front and back sides of the copper EAD antenna at its resonance frequency, $307 \mathrm{MHz}$.

Consequently, a quasi-isotropic conductivity is desired to achieve a satisfactory antenna performance. However, due to the minimum feature resolution of the embroidered threads ( $0.25 \mathrm{~mm}$ ), it is difficult to achieve an accurate dimensionality and the designed stitching patterns to allow the threads to achieve a good level of quasi-isotropic behavior. Consequently, slightly worse performance was expected from the embroidered EAD antenna.

For the purpose of defining the $k a$ values and the related quality factors of the three antennas, the overall electrical volume occupied by the EAD antennas can be considered to be the maximum size of the conducting features. For each case, the antenna's diameter: $147.30 \mathrm{~mm}$, was taken as this value. Thus, the antennas are electrically small with $k a=$ 0.474 at their resonance frequency, $307 \mathrm{MHz}$.

The simulated 3D gain pattern predicted for the copper shim version of the EAD is illustrated in Fig. 7. The corresponding simulated $\mathrm{E}$ and $\mathrm{H}$-plane polar plots of all three designs are given in Fig. 8 at their resonance frequencies.

As expected for an electric type ESA, these E-plane realized gain patterns are typical of an electric dipole antenna. On the other hand, the corresponding H-plane patterns are not purely azimuthally symmetric. This is due to the asymmetric current distributions around the conducting elements of the antenna corresponding to the electric field distributions depicted in Fig. 6. In particular, the high current densities on the edges of the capacitive arms of the EAD NFRP element and the presence of the balun are the main causes of this slight asymmetry. The simulated realized gain patterns presented in Fig. 8 also show that the radiated field is highly symmetric with respect to both the $\mathrm{E}$ - and H-planes. This behavior is indicative of the fact that the driven element has little effect on the overall radiation characteristics of the EAD antenna. This behavior is a known property of these NFRP antennas [22].

The realized gain of the antennas is immediately associated with the degree of conductivity of the material used to create the NRFP EAD element. The maximum simulated gain $\left(\mathrm{G}_{\max }\right)$ and the corresponding radiation efficiency (RE) values of the three EAD antennas were: copper shim EAD NFRP, $\mathrm{G}_{\max }=$ $1.41 \mathrm{dBi}$ with $\mathrm{RE}=91 \%$; copper veil EAD NFRP, $\mathrm{G}_{\max }=1.05$ $\mathrm{dBi}$ with $\mathrm{RE}=85 \%$; and the embroidered $\mathrm{EAD}$ NFRP, $\mathrm{G}_{\max }=$ $0.88 \mathrm{dBi}$ with $\mathrm{RE}=81 \%$ radiation efficiency. Simulations of the radiation patterns for the embroidered and copper veil NFRP EAD antennas also indicated a higher degree of ripple in the H-plane radiation pattern than present with the copper shim version.

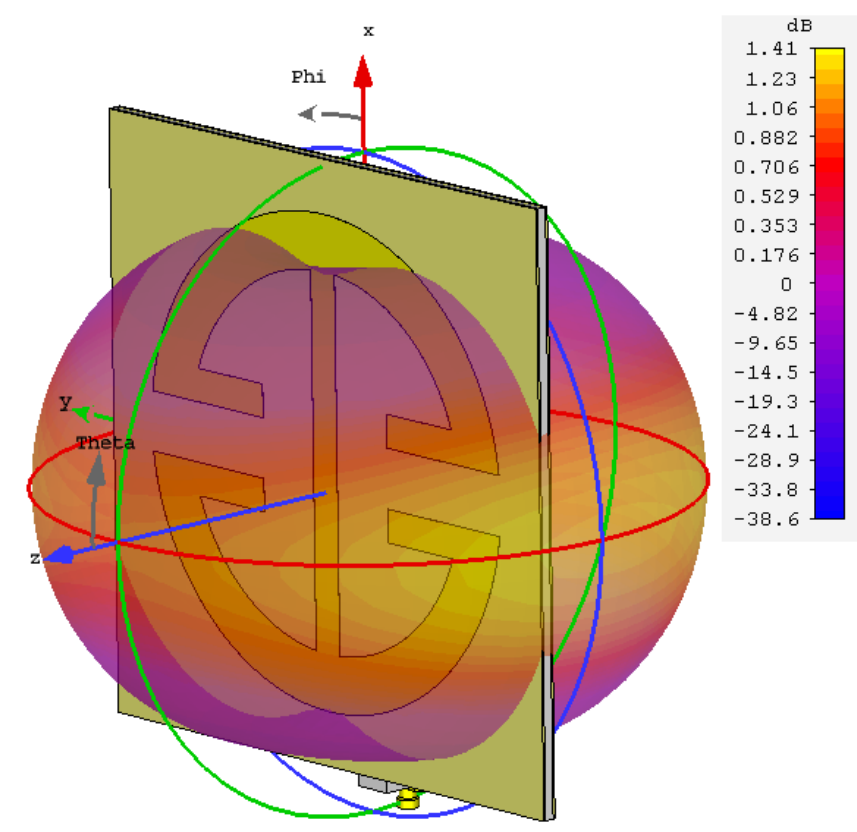

Fig. 7. Simulated $3 \mathrm{D}$ gain pattern of the copper EAD at its resonance frequency, $307 \mathrm{MHz}$. 


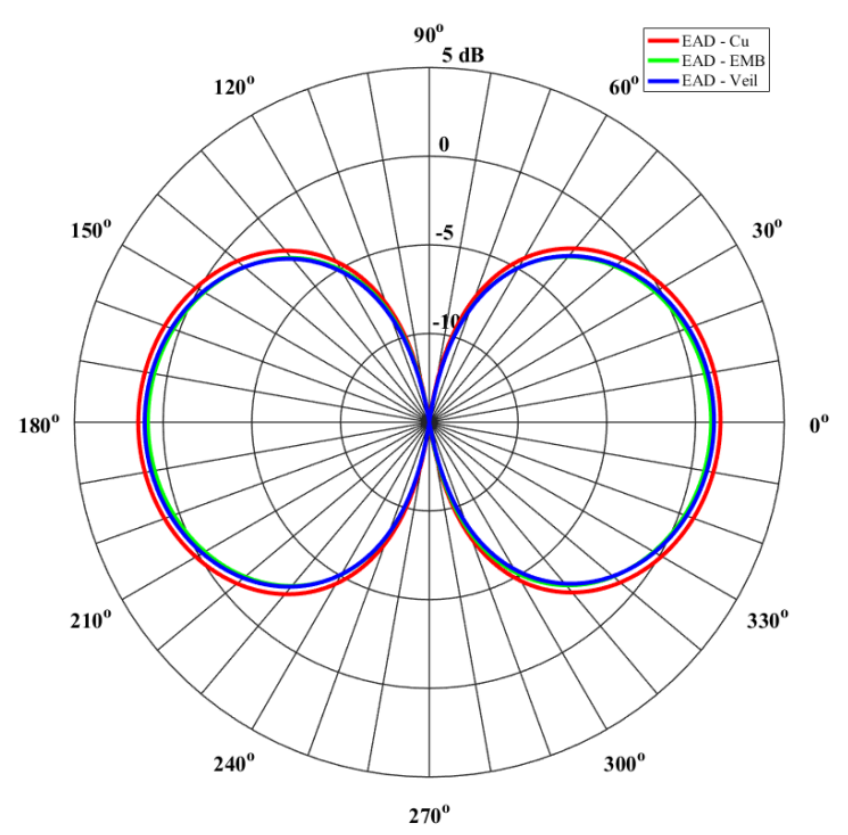

(a)

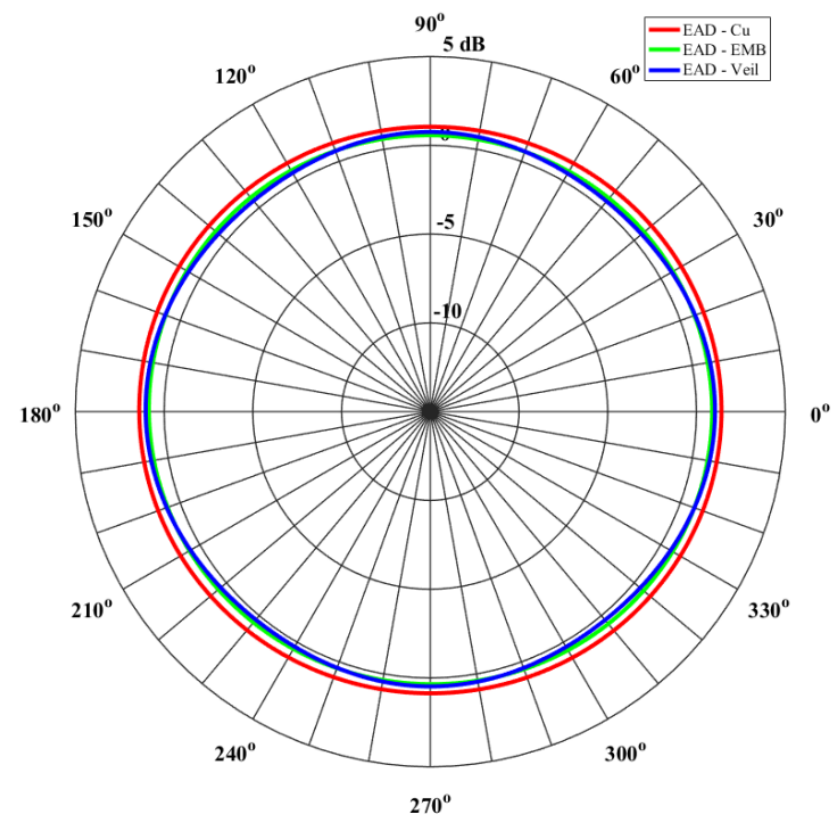

(b)

Fig. 8. Polar plots of the simulated a) E-plane and b) H-plane realized gain patterns for all three antennas at their resonance frequency, $307 \mathrm{MHz}$.

This ripple is upwards of $0.5 \mathrm{~dB}$ in both materials, which is mainly due to the small current distortions that exist around the EAD NFRP and driven elements. In hind sight, it could have been minimized if tapered corners had been used in the meander elements. The overall performance of the simulated EAD antennas showed reasonable gain in the presence of the new materials and their patterning techniques.

\section{Mechanical Design}

The stiffness of a composite sandwich core structure can be analyzed using the sandwich panel theory [25]. The stiffness of the proposed sandwich structure can be evaluated based on its flexible rigidity / panel bending stiffness ' $\mathrm{D}$ '. The flexible rigidity for the sandwich panel is defined as:

$D=E_{f} \frac{b t_{f}{ }^{3}}{6}+E_{f} \frac{t_{f} h^{2} b}{2}+E_{c} \frac{b t_{c}{ }^{3}}{12}$

where ' $E_{f}$ ' is the modulus of elasticity for the facing skin material, ' $\mathrm{E}_{\mathrm{c}}$ ' is the modulus of elasticity for the core material, ' $t_{c}$ ' is the core thickness, ' $t_{f}$ ' is the facing skin thickness, ' $h$ ' is the total panel thickness, and ' $b$ ' is the panel width. The loading condition typically seen by sandwich panels causes the middle term in Eqn. 1 to be dominant. Therefore, the flexible rigidity can be evaluated as:

$D=E_{f} \frac{t_{f} h^{2} b}{2}$

The shear stiffness of the panel can also be determined as:

$S=b h G_{c}$

where ' $\mathrm{G}_{\mathrm{c}}$ ' is the shear modulus of the core. The deflection induced in the panel for a given load can be evaluated by adding the flexible rigidity and the shear stiffness:

$\delta=\frac{k_{b} P l^{3}}{D}+\frac{k_{s} P l}{S}$

where ' $\mathrm{k}_{\mathrm{b}}$ ' and ' $\mathrm{k}_{\mathrm{s}}$ ' are the bending and shear deflection coefficients, respectively; ' $\mathrm{l}$ ' is the panel length; and $\mathrm{P}$ is the applied force. For a simply supported panel under a distributed load, $\mathrm{k}_{\mathrm{b}}=5 / 384$ and $\mathrm{k}_{\mathrm{s}}=1 / 8$. Furthermore, the applied force resulting from a distributed load ' $\mathrm{q}$ ' is:

$P=q l b$

Finally, utilizing equations (1) through (5), a load-bearing EAD antenna can be designed to suit a variety of mechanical load conditions. However, for the near-term purposes of this work, the typical load conditions endured, for example, by a small UAV are easily handled by the Astroquartz and Rohacell foam core.

\section{EAD MANUFACTURING PROCESS}

The EAD antennas were first manufactured on a dry Astroquarts II high purity fused silica fabric. It was then infused with the Lonza PT-30 cyanate ester resin. A cyanate ester resin system was selected for use as it has one of the lowest loss-tangents of all common high strength composite resins (such as epoxy, phenolic, BMI, etc.). Coupled with the high purity fused silica, a composite material emerges that exhibits very good RF performance, as was discussed in Section II. 


\section{A. Embroidered EAD (EEAD)}

The pattern of the EAD was sewn using a Brother PE-1000 computer controlled embroidery machine. The stitching pattern was manually generated within the Brother PE-Design Next software package. The stitch pattern of the EAD was carried out in three stages. The first stage consisted of sewing a $10 \mathrm{~mm} \times 10 \mathrm{~mm}$ grid pattern into the nine plies of the Astroquarts II fabric using a conductive Kevlar thread. This grid pattern was required to reduce the fabric distortions during the embroidery process with the conductive threads.

The second stage involved sewing a zigzag stitch over the NFRP EAD structure using a bobbin stitch approach [19]. This stage forms cross-threads so that the embroidered pattern achieves a quasi-isotropic conductivity. Unlike traditional needle stitching, the bobbin stitch approach allows for a high conductivity to be achieved [19]. The bobbin thread was replaced with the Aracon XS0200E-025 thread, while the needle thread was made from Kevlar, to sew the EAD antenna.

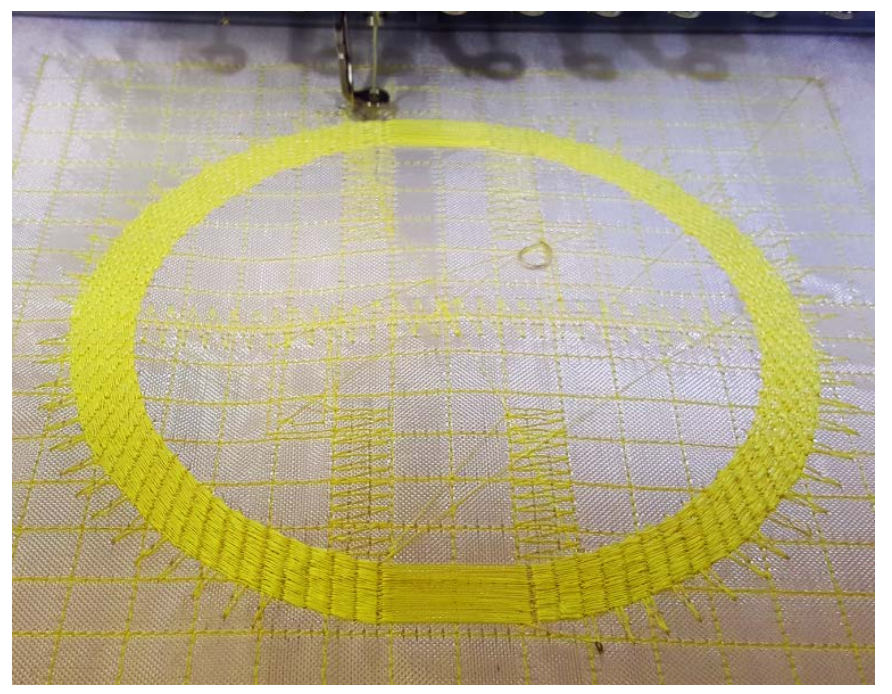

Fig. 9. Embroidery of the EAD dipole on the AstroQuartz II fabric. The yellow color of the dielectric Kevlar needle thread is shown. The Aracon conductive thread is sewn on the underside of the fabric.

The final stage of the embroidery of the EAD antenna was to lay down the Aracon thread in a radial stitch pattern over the zigzag stitch. Both the straight inner dipole and its curved capacitive ends were sewn using a running stitch to maximize the current flow along the NFRP element, as it would be in the copper version. All stitching patterns were sewn with a running pitch of $3 \mathrm{~mm}$ and a stitch density of 5 lines $/ \mathrm{mm}$ (or higher where required). An illustration of the embroidery process for the NFRP EAD element is shown in Fig. 9.

\section{B. Laser Cut Veil and Copper shim NFRP EAD}

The embroidery of the conductive threads has a number of drawbacks from a structural perspective. It has been shown in [20] that the embroidery process reduces the load carrying capacity of a composite part. This is due to the thick conductive threads causing localized damage and forming small defects within a cured composite structure. From an electrical perspective, the thread is also a highly anisotropic material and, as a result, an overlapped cross-stitch pattern is required to provide some level of isotropy. Consequently, a large bulk of conductive thread is accumulated on the quartz fabric, which needs to be consolidated within the thin laminate. This is a troublesome aspect of the process and can result in laminate bulging and, hence, very poor performance of the resulting antenna.

To simplify and improve the functionality of building conformal load bearing antennas, a new conductive veil concept is introduced here. This material consists of a copper coated, non-woven carbon veil. The carbon veil is lightly seeded in nickel and then electro-plated in high purity copper to form a complex jacket over the chopped-stand carbon fiber. Plating times were varied to achieve a layer plating thickness of 7 to $9 \mu \mathrm{m}$ over the carbon fibers.

The coated sheets were then treated with Silane to mitigate corrosion and oxidation whilst improving adhesion to the resin system. The treated material was then patterned using a computer controlled high power $\mathrm{CO}_{2}$ laser cutter (84 Watts). An illustration of the laser patterning of the EAD structure from the veil material is shown in Fig. 10. It should be noted that $\mathrm{CO}_{2}$ lasers do not cut metals very well due to their low emissivity. However, with the inclusion of the non-woven carbon fiber veil and the corrosion treatment solution, the laser was easily able to penetrate the thin copper jacket and cut the material precisely, leading to a very accurate realization of the EAD antenna design.

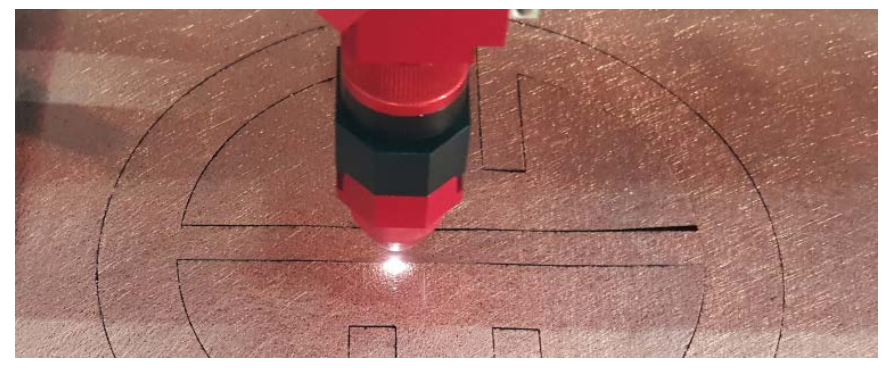

Fig. 10. Cutting of the $\mathrm{EAD}$ shape using a $\mathrm{CO}_{2}$ laser

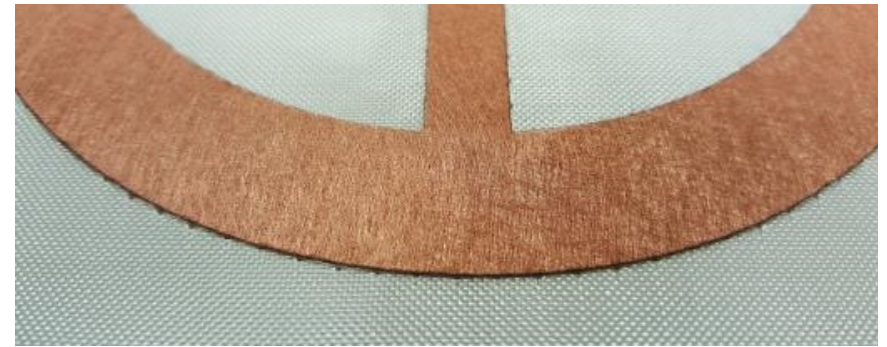

Fig. 11. Transferred EAD veil structure on to the Astroquarts II fabric

The laser slightly damaged the fibers around the cutting area due to the high heat generated locally from the laser spot. This damage occurred in a region on the order of $50 \mu \mathrm{m}$, which was insignificant for the $300 \mathrm{MHz}$ (1.0 m wavelength) design frequency of the EAD antenna. Prior to transferring the veil structure onto the Astroquartz II, the fabric was prepared 
using embroidery. The outline of the EAD antenna as well as its $180 \mathrm{~mm}$ x $180 \mathrm{~mm}$ boarder was stitched onto nine plies of the Astroquartz II material using the Kevlar thread. The copper veil structure was then weakly adhered with a contact adhesive. An image of the positioned EAD structure on the Astroquartz II fabric is depicted in Fig. 11. The outlining embroidery is just visibly observed at the edge of the veil material.

The copper reference EAD antenna was manufactured in a similar way to the veil, with the exception of the EAD element which was micro-machined on an LPKF PCB mill. The EAD antenna was made with a $100 \mu \mathrm{m}$ high purity copper shim, which was weakly adhered to a FR-4 substrate. Once machined on the LPKF mill, the EAD elements were lifted using a solvent and then cleaned, coated, and finally transferred onto the Astroquartz II fabric. The latter was embroidered with the outline of the EAD element of guarantee correct positioning of all of the components.

\section{EAD Driven Element}

The driven element was also micro-milled using the high purity $100 \mu \mathrm{m}$ copper shim. Its manufacturing process was identical for all three antennas. The milled driver element was joined at the center to allow for easy transfer. The outline of the NFRP element was again embroidered into nine plies of the Astroquarts II fabric. The milled copper sections were transferred and weakly adhered to the fabric. After consolidation and curing of the QFRP material, a feeding gap of $0.508 \mathrm{~mm}$ was cut through the copper to allow for the correct feeding arrangement of the balun.

\section{Completed Assembly of the EAD Antennas}

All of the EAD structures (copper shim, embroidered and copper veil) were consolidated, infused with resin, and cured in an autoclave at $200^{\circ} \mathrm{C}$ at $700 \mathrm{kPa}$ pressure as recommended by the Lonza PT-30 cyanate ester resin datasheet. The thin cyanate ester substrates were then roughly trimmed and their back face roughened in preparation for bonding to the Rohacell HF foam core. To bond the substrates to the foam core, a small amount of the Lonza PT-30 cyanate ester was used. The driving element substrate was firstly bonded onto the foam core and cured at $140^{\circ} \mathrm{C}$ under vacuum for six hours. Post bonding, the feed gap was machined and the balun was soldered into position. The EAD NFRP substrate was then adhered using the cyanate ester resin to the driving element and foam substrate. However, prior to curing, the tuning of the EAD was checked. At room temperature $\left(\sim 23^{\circ} \mathrm{C}\right)$, the cyanate ester resin is extremely viscous and sticky (with a consistency thicker than honey). Using a calibrated Keysight $10 \mathrm{MHz}-50$ $\mathrm{GHz}$ N5225A PNA analyzer, the input impedance and matching of the antenna was tested. During this process, the alignment of the two surfaces was also carried out to ensure optimum impedance matching and antenna efficiency. The viscous state of the cynate ester resin held the tuned position of the EAD elements while the substrate was secured using high temperature flash-breaker tape. The balun was then desoldered and the aligned antenna was placed back into an oven

under vacuum for a further 24 hours to complete the bonding process. The final processing step included trimming the substrate. The balun was then reattached with the inclusion of its Rohacell HF supporting block. Both were glued into position using a small amount of quick drying super glue. An illustration of the three assembled antennas is given in Fig. 12. It should be noted that the use of a fabric as the substrate material allows for these structures to be cured into a conformal shape. Both the embroidered and veil versions have sufficient drapability to be formed around a double-curvature conformal surface, such as an airfoil or car side panel, while the copper version is only suited for well-defined 2D surfaces such as planar and cylindrical shapes. Both materials also conform well to traditional composite manufacturing processes [26].

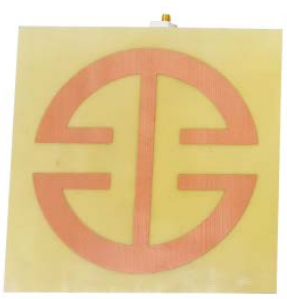

a)

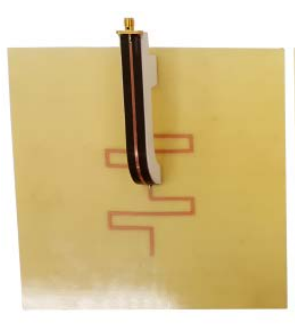

Fig. 12. Illustration of the top and bottom sides of all three fabricated EAD antennas: a) copper shim, b) embroidered, and c) copper veil.

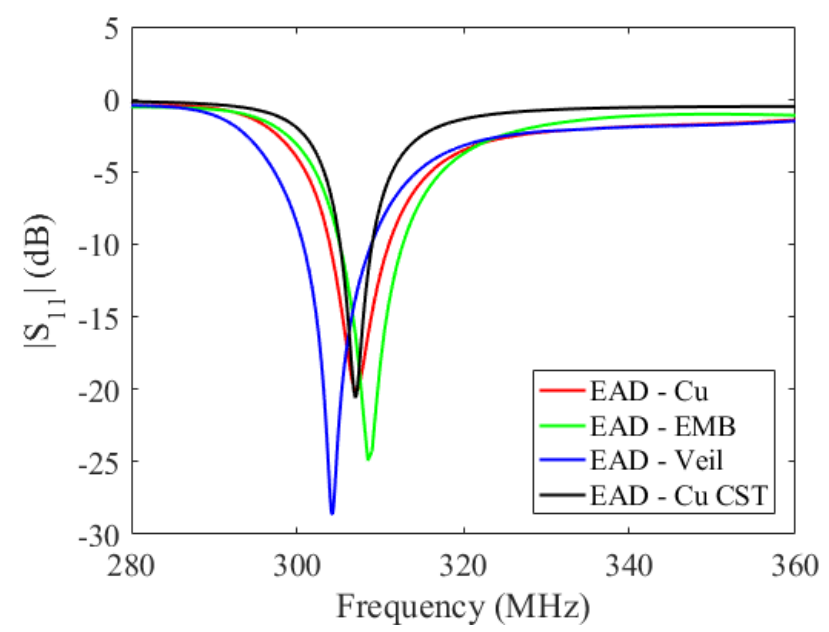

Fig. 13. Measured $\left|S_{11}\right|$ values as a function of the source frequency for all three EAD composite antennas compared with the simulated values for the copper shim version. 


\section{EAD ANTENNA PERFORMANCE}

The magnitudes of the reflection coefficient, $\left|S_{11}\right|$, for all three EAD antennas were measured with a SOLT calibrated Keysight N5225A PNA analyzer. The calibration and measurement were carried out within a semi-anechoic chamber. The measured $\left|S_{11}\right|$ results are illustrated in Fig. 13 as functions of the source frequency. As demonstrated, all three manufactured antennas are very well matched at the approximate design frequency.

The slight deviation between the resonance frequency of the embroidered and veil antennas is due to a small dimensional mismatch in the antennas' EAD NFRP or driven elements and/or the consolidated thickness of the QFRP laminate material. The value of $\left|S_{11}\right|_{\text {min }}$ for the copper shim EAD compared to the simulated ideal copper case (EAD - CuCST) indicates very good agreement. With the exception of the resonance shift, the embroidered and veil antennas are also showing very good agreement with their simulated results.

It should be noted that for the evaluation of these $307 \mathrm{MHz}$ antennas, this measurement arrangement was not ideal because of the actual size of the chamber and the absorber cones in it. There was an expectation that some level of radiation would interact with the chamber structure itself and the net effect would slightly corrupt the results. While conducting the measurements in this semi-anechoic chamber reduces the likelihood of coupling to the cavity modes of the chamber walls, there still is some distortion within the radiation patterns measured for the EAD antennas. The measured radiation patterns were all obtained relative to a pair of matching $306 \mathrm{MHz}$ monopole antennas. These monopole antennas were all standardized against a Singer DM-105A-T2 $140 \mathrm{MHz}$ to $400 \mathrm{MHz}$ tunable dipole antenna.

The gain patterns for the EAD antennas were also measured within the same semi-anechoic chamber. The measured E- and $\mathrm{H}$-plane gain patterns for all three EAD antennas are presented in Fig. 14. Significant distortions within the radiation patterns are clearly visible in both the E- and H-planes patterns. They are a result of a right angle SMA cable traversing the expected pattern null directions which, unfortunately, was required when feeding the antenna in the semi-anechoic chamber. The E-plane gain patterns most notably illustrate the consequent removal of the underside null. This direction is the location of the balun and the SMA connector feeding it.

Observing the shape of the E-plane realized gain patterns from 0 to 180 degrees, away from the cable issues, there is good conformability to the expected simulated patterns. A slight skewing of the pattern is seen in both the $\mathrm{E}$ and H-plane plots, which suggests that the alignment of the EAD NFRP and driven elements was not perfect, despite the extra tuning stage. This alignment for composite structures is much more difficult to achieve than using non-structural, traditional materials, because of the fluid nature of the resin during the curing process. This is one aspect of the composite manufacturing process that requires further investigation to refine its repeatability, especially when arbitrary conformal structures are desired.

The H-plane gain patterns also show good agreement with their simulated ones. It should be noted that the distortion in the E-plane is not seen in the H-plane results since the feeding cables transfer their energy parallel to the balun; and, hence, there is no missing section present in these measurements. A direct comparison of all of the gain values with their simulated results is difficult to make due to the distorted patterns. In all cases, the peak gain of each EAD antenna is greater than the reference dipole antenna. However, the pattern ripple is significant, again due to the measurement arrangement. Keeping in mind the distortions and the good agreement of the nulls in the $\left|S_{11}\right|$ values, the antennas appear to be radiating with or close to their expected performance characteristics.
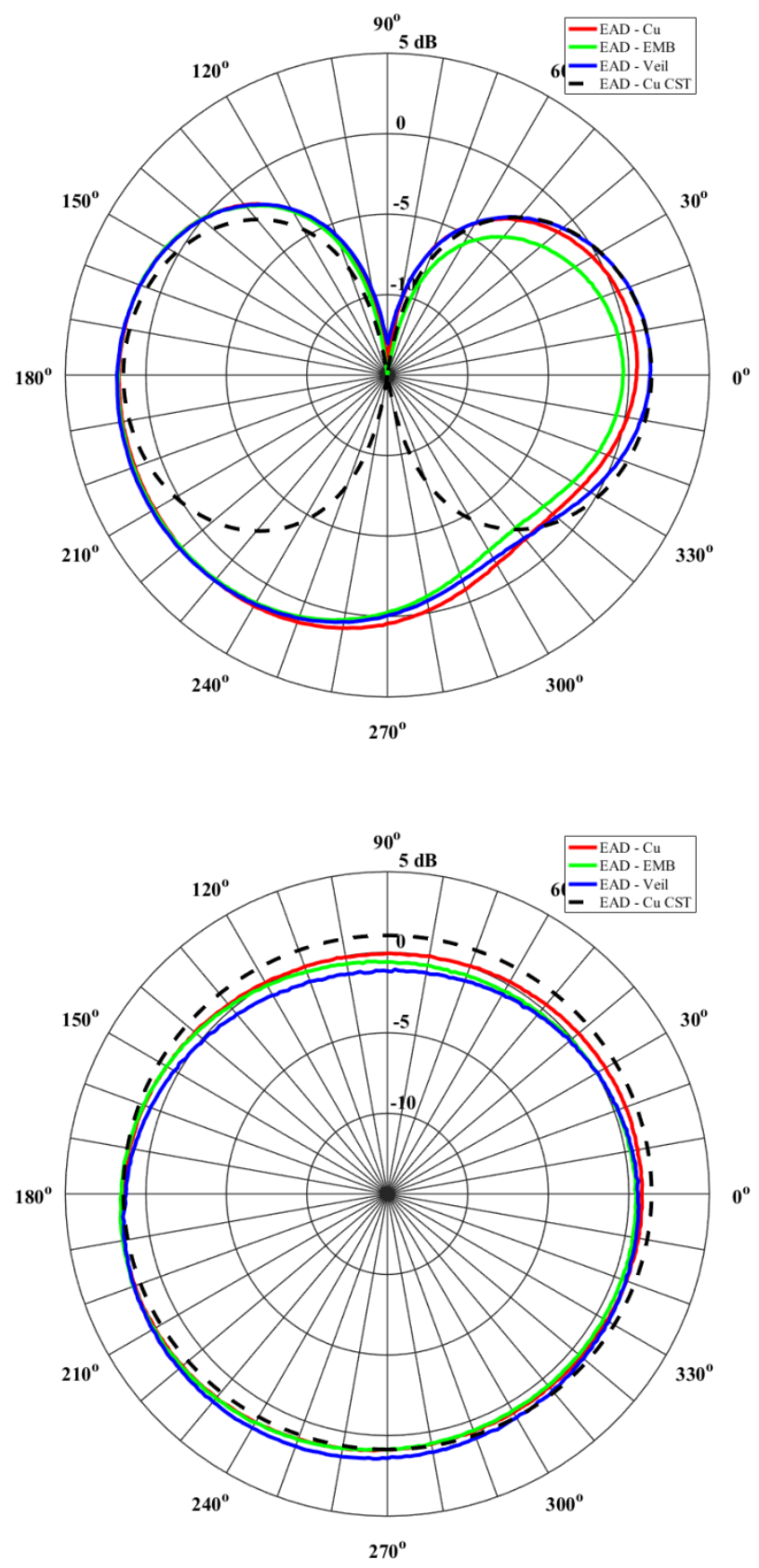

Fig. 14. Measured and simulated E-plane (top) and H-plane (bottom) realized gain patterns for the $\mathrm{EAD}$ antenna. 
Table I gives a summary of all the measured antenna performance characteristics. All of the values are very close to one another. The veil case exhibited a maximum gain much higher than the embroidered case as expected.

The maximum gain value, $G_{\max }$, of the veil case is higher and the corresponding minimum value, $G_{\text {min, }}$ is smaller than that of the copper case. This is most likely due to the pattern distortion introduced by the measurements. The radiation efficiency $\left(\eta_{\text {radiation }}\right)$ values are all very good, hence, the bandwidth (BW) values (quality factors) are not large (small) simply because of losses. Given that the $k a=0.4$ value of the measured veil case is the smallest, these outcomes are very attractive for a number of electromagnetic devices embedded in structural material applications, including communication systems, sensors, and remote sensing devices.

The mass of the antennas has also been included in Table I. The variance in the mass of each antenna indicates the accumulative difference from the conducting materials used in the EAD NFRP element and the amount of cyanate ester resin cured within the QFRP laminates. All other structural elements are identical in volume and approximate weight. The mass difference of the conducting Aracon thread and carbon veil is very similar. Therefore, the majority of the observed mass can be attributed to the cyanate ester resin. The mass of the veil antenna indicates it contains more resin than the copper and embroidered EAD antenna. Considering the measured $\left|S_{11}\right|$ values in Fig. 13, the slight shifts in the resonance frequencies may also be attributed in part to the increase or decrease in the amount of resin used in their construction.

When visibly observing the EAD antennas in Fig. 12, their color follows the same trend (veil, copper shim, and then embroidery). A darker color indicates the accumulation of more cyanate ester resin within the QFRP laminate. The accumulation of the extra resin within the veil QFRP laminates will increase the thickness of the laminate; hence a downward shift in the resonant frequency is expected. The opposite trend is expected for less resin such as that seen in the embroidered EAD. The same trend is observed in Fig. 13.

TABLE I

Summary of THE MEAsured Performance Characteristics of the EAD ANTENNAS AND THEIR STRUCTURAL PARAMETERS

\begin{tabular}{|l|l|l|l|}
\cline { 2 - 4 } \multicolumn{1}{c|}{} & EAD - Cu & EAD - EMB & EAD - Veil \\
\hline $\mathrm{f}_{\mathrm{r}}(\mathrm{MHz})$ & 307 & 308.5 & 304.25 \\
\hline $\mathrm{BW}-{ }_{10 \mathrm{~dB}}(\mathrm{MHz})$ & 7.2 & 6.6 & 8.13 \\
\hline$\eta_{\text {radiation }}(\%)$ & 91 & 81 & 85 \\
\hline $\mathrm{Q}_{\text {Chu }}$ & 11.53 & 11.44 & 11.80 \\
\hline $\mathrm{G}_{\text {max realized }}(\mathrm{dBi})$ & 1.72 & 1.79 & 1.9 \\
\hline $\mathrm{G}_{\min \text { realized }}(\mathrm{dBi})$ & -0.12 & -0.68 & -1.22 \\
\hline $\begin{array}{l}\mathrm{G}_{\max } \text { realized CST } \\
(\mathrm{dB})\end{array}$ & 1.41 & 0.88 & 1.05 \\
\hline Mass $_{\text {total }}(\mathrm{g})$ & 74.22 & 57.99 & 84.99 \\
\hline
\end{tabular}

\section{CONCLUSION}

This work investigated three EAD antennas manufactured using a composite sandwich-structured material consisting of two structural QRFP substrates surrounding a foam stiffened core. Two of the reported antennas were constructed with novel conductor materials, an embroidered type and a carbon veil type, which use different design and fabrication processes. The design and manufacturing processes provided a unique way to seamlessly integrate CLAS antennas into structural composite materials. All of the measured antenna results positively demonstrated the ability to realize structural loadbearing electrically small antennas with satisfactory performance characteristics. This EAD antenna is well suited for wireless applications associated with UAVs in which many mechanical- and aerospace-purposed structures are manufactured entirely from glass/quartz. These include winglets and wings, as well as vertical and horizontal stabilizers. Actual antenna locations would be selected to take advantage of its omni-directional radiation pattern.

In the evaluated designs, the inclusion of low conductivity materials caused an overall decrease in gain; however, this was within acceptable limits. The veil case, as expected, performed very well in comparison to the reference copper version. The loss issue was illustrated with the embroidered version. Nonetheless, the radiation performance of both the embroidered and the veil EAD antennas indicates that both the embroidery and the veil materials and their processing techniques are suitable for developing ESAs in composite materials. For the embroidery process, more research is required to develop stitching patterns that provide consistently some level of quasi-isotropic and higher conductivity values. Furthermore, as outlined in [19], the consolidation temperatures and pressures of high performance composite structures allows for better quasi-isotropic conductivity to be achieved with less threads, as the fibers are pushed together and then locked into the polymer matrix. This is unlike textiles-based embroidery where the threads typically make poor contact. On the other hand, the veil material has immediate applicability to achieve high performance, electrically small conformal antennas in modern loadbearing structural materials with applications to several types of mobile platforms including drones, land vehicles, aircraft and ships, as well as to modern architectural structures that could take advantage of embedded communication, wireless power transmission, and sensor networks.

\section{ACKNOWLEDGMENTS}

This work was supported by the Australian Department of Defence, Defence Science and Technology Group (DSTG) SRI-SME. The initial contributions by R. W. Ziolkowski to this work occurred when he was the 2014-2015 Australian DSTO Fulbright Distinguished Chair in Advanced Science and Technology. He would like to thank the AustralianAmerican Fulbright Commission and the Defense Sciences and Technology Group (formally DSTO) for their support. 


\section{REFERENCES}

[1] A. J. Lockyer, K. H. Alt, J. N. Kudva, R. W. Kinslow, and A. C. Goetz, "Conformal load-bearing antenna structures (CLAS): initiative for multiple military and commercial applications," in Proc. SPIE3046, Smart Structures and Materials 1997, San Diego, CA, Mar. 1997, pp. 182-196.

[2] M. Hopkins, J. Tuss, A. Lockyer, K. Alt, R. Kinslow, and J. Kudva, "Smart skin conformal load-bearing antenna and other smart structures developments," in Proc. 38th Structures, Structural Dynamics, and Materials Conference, American Institute of Aeronautics and Astronautics, Kissimmee, FL, Apr. 1997, pp. 521-530.

[3] P. J. Callus, "Novel concepts for conformal load-bearing antenna structure," Defence Science and Technology Organisation (DSTO), DSTO-TR-2096, 2008, pp. 1 - 111.

[4] H. S. Kim, J. S. Kang, J. S. Park, H. T. Hahn, H. C. Jung, and J. W. Joung, "Inkjet printed electronics for multifunctional composite structure," Composites Science and Technology, vol. 69, Issue 7-8. pp. 1256-1264, Jun. 2009.

[5] A. V. Kumar, "Multifunctional Composite Structures," DTIC Document AFRL-RW-EG-TR-2010-047, 2010, pp. 1 - 128

[6] K. J. Nicholson, W. S. T. Rowe, K. Ghorbani, and P. J. Callus, "Splitring resonator loaded miniaturized slot for the slotted waveguide antenna stiffened structure," in Proc. The Fifth International Congress on Advanced Electromagnetic Materials in Microwaves and Optics, Metamaterials, Barcelona, Spain, Oct. 2011, pp. 540-542.

[7] A. Bojovschi, D. Gray, and K. Ghorbani, "A loop-type end-launcher for carbon fiber reinforced polymer waveguides,” Progress In Electromagnetics Research M, vol. 31, pp. 13-27, 2013.

[8] R. McCauley, "Material design methodology for structural and microwave multifunctional composite laminate systems," Doctoral Dissertation, University of Delaware, 2013.

[9] J. W. Sabat and A. N. Palazotto, "Structural performance of composite material for a slotted waveguide antenna stiffened structure under compression," Composite Structures, vol. 97, pp. 202-210, Mar. 2013.

[10] L. J. Chu, "Physical limitations of omnidirectional antennas," J. Appl. Phys., vol. 19, pp. 1163-1175, Dec. 1948.

[11] H. A. Wheeler, "Fundamental limitations of small antennas," in IRE Proc., Dec. 1947, vol. 35, pp. 1479-1484.

[12] R. W. Ziolkowski, M.-C. Tang and N. Zhu, "An efficient, broad bandwidth, high directivity, electrically small antenna," Microw. Opt. Technol. Lett., vol. 55, no. 6, pp. 1430-1434, June 2013.

[13] N. Zhu and R. W. Ziolkowski, "Broad bandwidth, electrically small, non-Foster element-augmented antenna designs, analyses, and measurements," IEICE Transactions on Communications, vol. E96-B, no.10, pp. 2399-2409, Oct. 2013.

[14] H. Mirzaei and G. V. Eleftheriades, "A resonant printed monopole antenna with an embedded non-Foster matching network," IEEE Trans. Antennas Propag., vol. 61, no. 11, pp. 5363-5371, Nov. 2013.

[15] K.-M. Luk and B. Wu, "The magnetoelectric dipole: A wideband antenna for base stations in mobile communications," Proc. IEEE, vol. 100, no. 7, pp. 2297-2307, Jul. 2012.

[16] H.-J. Seo, and A. A. Kishk, "Wideband magnetic-electric antenna with linear single or dual polarization," Progress In Electromagnetics Research (PIER), vol. 155, pp. 53-61, Mar. 2016.

[17] M.-C. Tang, H. Wang, and R. W. Ziolkowski, "Design and testing of simple, electrically small, low-profile, Huygens source antennas with broadside radiation performance," IEEE Trans. Antennas Propag., vol. 64, no. 11, pp. 4607-4617, Nov. 2016.

[18] M.-C. Tang, T. Shi, and R. W. Ziolkowski, "Electrically small, broadside radiating Huygens source antenna augmented with internal non-Foster elements to increase its bandwidth," IEEE Antennas Wirel. Propag. Lett., vol. 16, pp. 712-715, 2017

[19] T. C. Baum, R. W. Ziolkowski, K. Ghorbani, and K. J. Nicholson, "Embroidered active microwave composite preimpregnated electronics Pregtronics," IEEE Trans. Microw. Theory Tech., vol. 64, pp. 31753186, Sep. 2016.

[20] K. Nicholson, O. Dunbabin, T. Baum, and K. Ghorbani, "Characterisation of integrated microstrip lines in aerospace composite structure,” Electron. Lett., vol. 53, no. 1, pp. 36-38, Jan. 2017.

[21] T. C. Baum, K. J. Nicholson, A. Galehdar, K. Ghorbani, and R. W. Ziolkowski, "Multi-functional composite metamaterial-inspired EEAD antenna for structural applications," Proc. International Workshop on Antenna Technology, iWAT 2016, Cocoa Beach, FL, Feb. 29-Mar. 2, 2016, pp. 144-147.
[22] R. W. Ziolkowski, P. Jin and C.-C. Lin, "Metamaterial-inspired engineering of antennas," Proc. IEEE, vol. 99, pp. 1720-1731, Oct. 2011.

[23] P. Jin and R. W. Ziolkowski, "High directivity, electrically small, lowprofile, near-field resonant parasitic antennas," IEEE Antennas Wireless Propag. Lett., vol. 11, pp. 305-309, 2012.

[24] W. B. Weir, "Automatic measurement of complex dielectric constant and permeability at microwave frequencies," Proc. IEEE, vol. 62, no. 1. pp. 33-36, Jan. 1974.

[25] H. G. Allen, "Chapter 2 - Sandwich Beams," in Analysis and Design of Structural Sandwich Panels, ed: Pergamon, 1969, pp. 8-47.

[26] A. A. Baker, S. Dutton and D. W. Kelly, Composite Materials for Aircraft Structures, 2nd Ed., American Institute of Aeronautics and Astronautics, Inc., Reston, VA, 2004.

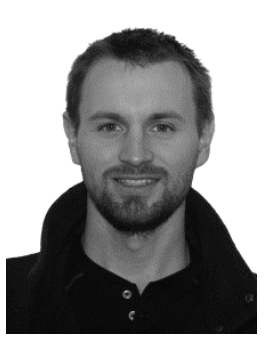

Thomas C. Baum (M'10) received his B.Eng in Aerospace Engineering (Hons.) at RMIT University in 2009. He completed his PhD. in electrical engineering for RMIT University in 2014. In 2015 he became a research fellow at RMIT University working on a variety of microwave related research areas including conformal load bearing antenna structures, coherent Doppler tomography and dielectric material characterization. His interests lie in novel antenna designs, radar systems, radar absorbing applications, scattering phenomena, microwave imaging, and material characterization.

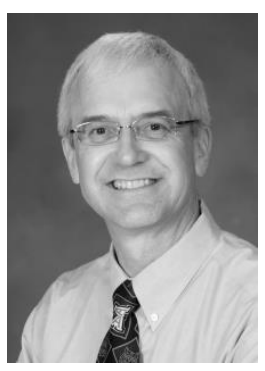

Richard W. Ziolkowski (M'87-SM'91F'94) received the B.Sc. (magna cum laude) degree (Hons.) in physics from Brown University, Providence, RI, USA, in 1974; the M.S. and Ph.D. degrees in physics from the University of Illinois at Urbana-Champaign, Urbana, IL, USA, in 1975 and 1980, respectively; and the Honorary Doctorate degree from the Technical University of Denmark,

Kongens Lyngby, Denmark in 2012.

He is currently a Distinguished Professor with the Global Big Data Technologies Centre, University of Technology Sydney, Ultimo NSW, Australia. He is also a Litton Industries John M. Leonis Distinguished Professor with the Department of Electrical and Computer Engineering and a Professor with the College of Optical Sciences, The University of Arizona. He was the Computational Electronics and Electromagnetics Thrust Area Leader with the Lawrence Livermore National Laboratory, Engineering Research Division, before joining The University of Arizona, Tucson, AZ, USA, in 1990. He was the Australian DSTO Fulbright Distinguished Chair in Advanced Science and Technology from 2014-2015. He was a 2014 Thomas-Reuters Highly Cited Researcher. His current research interests include the application of new mathematical and numerical methods to linear and nonlinear problems dealing with the interaction of electromagnetic and acoustic waves with complex linear and nonlinear media, as well as metamaterials, metamaterial-inspired structures, and applications-specific configurations. 
Prof. Ziolkowski is a Fellow of the OSA (Optical Society of America, 2006), and of the APS (American Physical Society, 2016). He served as the President of the IEEE Antennas and Propagation Society in 2005. He is also actively involved with the URSI, OSA and SPIE professional societies.

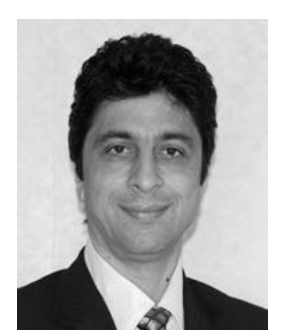

Kamran Ghorbani (M'96) received the B.Eng (Hons.) and Ph.D. degree from RMIT University, Melbourne, VIC, Australia, in 1994 and 2001, respectively. From Nov. 1994 to 1996, he was a graduate RF Engineer with AWA Defence Industries working on early warning radar systems. In June 1996, he joined RMIT University to pursue his Ph.D. studies. From Dec. 1999 to March 2001, he was a Senior RF Engineer with Tele-IP working on VHF transceivers for commercial aircraft. He joined the Department of Communication and Electronic Engineering (now the School of Electrical and Computer Engineering) at RMIT University in 2001 as a continuing Academic.

Prof. Ghorbani is currently the Director of the Communication Technologies Research Centre, RMIT University. He is responsible for strategic planning and managing the research center. His research interests include, dielectric measurements, composite material structures, frequency selective surfaces, metamaterials, RF energy harvesting, radar systems, ferroelectric devices and multifunctional antennas.

Prof. Ghorbani was the Chair of Asia Pacific Microwave Conference APMC2011, which was held in Melbourne, Australia. He was the Co-Chair of Technical Program Committee for IEEE International Microwave and RF Conference, IMaRC2014, which was held in India. He was the
Chair of first Australian Microwave Symposium, AMS2014, which was held in Melbourne, Australian. He is the Chair of Technical Program Committee of the Asia Pacific Microwave Conference APMC2016, which is going to be held in India. He is a member of IEEE MTT-S Meetings and Symposia Committee (AdCom sub-committee).

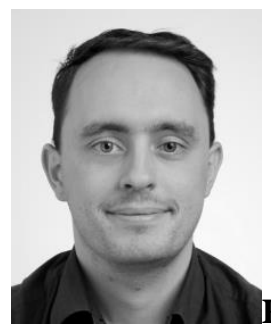
B.Sc in Physics and B.Eng (Hons.) in Electrical Engineering from the University of Melbourne in 2005. He joined the Defence Science and Technology Organisation (DSTO) in 2006 as part of the Aerospace Composites Technology group within the Aerospace Division. As part of this group, Kelvin has been involved in the development of microwave Doppler tomography imaging techniques for the assessment of damaged and repaired composite structures. He has assisted with the mitigation of electromagnetic interference issues aboard the ANZAC frigates and was awarded a Chief Defence Scientist Commendation in 2011 and a Royal Australian Navy Commendation in 2012. Kelvin completed his $\mathrm{PhD}$ in Electrical Engineering from RMIT University in 2014 with the support of the DSTO Enabling Research Program. His current research interests at DSTO include metamaterials and advanced composite materials for antenna and radar absorbing applications. 